Military Technical College

Kobry El-Kobbah, Cairo, Egypt.

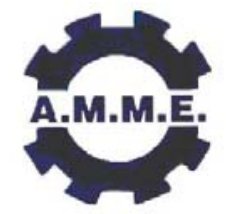

$13^{\text {th }}$ International Conference on Applied Mechanics and Mechanical Engineering.

\title{
FEATURE RECOGNITION TECHNIQUE FOR TURNING OPERATION
}

\author{
EL-HAKIM ${ }^{*}$ M.A. , AFIFI ${ }^{* *}$ A.A. and EL-MESALLAMY ${ }^{* *}$ A.S.
}

\begin{abstract}
The paper presents a new feature recognition technique which is considered the first module of an implemented computer aided process planning system [CAPP] for turning parts. The developed feature recognition technique involves the reconstruction of the part design features (lines, arcs), which are extracted from the CAD file in the form of manufacturing features (16 external features, and 10 internal features). It includes also retrieval of important data related to the created features (dimensions, tolerances and roughness) to be an efficient tool in process planning. Output data are displayed to the user as design features, and manufacturing features with all required data technique for manufacturing. A case study is given to demonstrate, and verify the proposed algorithm.
\end{abstract}

\section{KEY WORDS}

Feature Recognition, CAPP, CIM.

* Professor, Dpt. of Mechanical Engineering, Ain-Shams University, Cairo, Egypt.

** Assist. Prof., Dpt. of Mechanical Engineering, Modern Academy, Cairo, Egypt.

*** Egyptian Armed Forces. 


\section{INTRODUCTION}

Computer aided process planning [CAPP] system plays a key role in the integration of computer aided design (CAD), and computer aided manufacturing (CAM)[1], and hence in computer integrated manufacturing (CIM). Feature recognition is one of the most important stages of a CAPP system, as It reconstructs the product in the form of manufacturing features from the given design entities [2, 3, 4]. Researches in the field of CAD, and CAM are develop independently .CAPP in the context of an integrated information flow are still regarded as a weak point, which represents a vast field of research [3]. According to the survey of the different CAPP systems (FCAPP/SAMGEOPDE-GFAS-GLM-IKOOP-KAPLAN-Kbase-MCOES-PERMIA-RDCAPP-ROBEX and RATE-SMT-TAB-TAMCAM-TVCAPP-ICAPP-AutoCad MCAM) [3,6,7,8,9,10,11], it has been found out that there still exist certain points, which have not been covered sufficiently. Flexibility of the most systems is considerably low due to few numbers of predefined features, consideration of important technological constraints such as surface roughness, and tolerance.

In the present work, 2D CAD models are used for the determination of the necessary machining steps required to manufacture the object by turning on a general purpose center lathe. The first step is interfacing CAD to CAM, which is not a trivial problem since design features are in general quite different from manufacturing features and there is no easy way to translate a part description directly from the design domain to the manufacturing domain [5]. Turned parts have usually a large number of features. The feature recognition problem is not only to recognize the individual features of the workpiece but also to recognize the different combinations among them, and to extract right dimensions, tolerances and roughness. Increasing the extracted features (16 external, and 10 internal) makes the algorithm an efficient tool in a computer aided process planning system.

\section{Feature}

A feature is a combination of geometric entities (lines, arcs ...etc.), which constitutes the shape of an element of a given object represented by its working drawing [5].

\section{Feature Recognition}

It is a computer aided technique used to create features from geometric entities data structure of the part drawing (2D-CAD models) to match them with classified features, well known in manufacturing, using a rule based approach. Feature recognition involves retrieval of each feature data in the CAD models including dimensions, tolerances and roughness to reconstruct the part design based features, with a manufacturing based features. There are two main kinds of outputs of this module:-

1- Design entities which consist of lines, and arcs of the input part drawing with their extracted data such (start, and end points of a line - start, end, and angles of an arc - layer of design entities "external , internal").

2- Manufacturing features with the required manufacturing data (manufacturing dimensions "depth of cut, length of cut ", tolerances and roughness). 


\section{SYSTEM REQUIREMENTS}

The software program is developed to work under Microsoft windows 98 operating system or later. The main software program consists of several modules, which are developed under different programming and drafting AutoCAD package to be used for drafting of input part drawing. Visual basic 6 package has been used for constructing the system engine, and user interfaces (input and output).

\section{SYSTEM CONSTRAINTS}

The following rules have been considered in introducing the workpiece drawing to ensure consistent results from the system software. The workpiece coordinates zero point $P(0,0)$ should lie at the center right hand side of the part drawing as shown in Fig. 1 The part drawing should be a full crossectional view of the workpiece. External and internal features are to be drawn in two separate layers. The dimensions, tolerances and thread text formats must be drawn using associative dimensioning commands as shown in Fig. 2 The system will not consider the ordinary text as dimensioning entities. The input part drawing has to be drawn "to scale" with any arbitrary scale.

\section{Drawing Scale}

The user can input workpiece drawings with any arbitrary scale. The created system allows the user to enter the scale value through the form shown in Fig. 3. The output features will be in true dimensions.

\section{ENTITIES EXTRACTION MODULE}

Data exchange file DXF is the data file for the input part drawing. It consists of a group of lines, which contain all data of part drawing entities. The number of lines in the DXF file varies with the changes of the drawing entities. The DXF file consists of the following sections: Header section - Classes section - Tables section- Entities section Blocks section- Objects section. The entities section contains the graphical data included in the part drawing. The graphical data that will be extracted from the entity section to be used for the creation of the features are the start and end points of straight lines, center point, radius, starting angle, and end angle for arcs. This software performs a search in the DXF file to find out the start of the entities section. According to the entity type (lines or arcs) the search path will diverge. If the entity type is a straight line the search will continue to find the $(x, z)$ coordinates of the start and end points. On the other hand, if the entity is an arc the search will continue to find the center point, radius, starting angle and end angle respectively. This search methodology will be repeated till the end of the DXF file. All the search results will be sorted in a separate form.

\section{Data Preparation}

Although the data for all entities included in the full sectional view is given as shown in Fig.4-a, the feature creation module needs only the entities data of the half sectional 
view shown in the Fig.4-b. A certain logic is established for transforming the data associated with full sectional view into half sectional view as follows:

\section{1- Removing half section.}

The data belonging to the half section, lying above the horizontal line passing through the zero point will be removed from the extracted drawing data, while the data belonging to the half sectional view shown in Fig.5 will remain in the entities data.

\section{2- Removing the center line}

After executing this step the center line will be deleted from the entities saved data.

\section{3- Recognition of lines and arcs layers (external or internal)}

During this procedure the software will distinguish between external and internal lines and arcs by recognizing the layer of each entity in the DXF file.

\section{4- Removing non-required Parts of internal lines}

After specifying the internal features, some internal lines as shown in Fig.6 are not required for feature identification, and should be removed from the read data

\section{5- Recognition of line type (normal or polyline)}

Line type (normal or polyline) is recognized during this procedure for both external, and internal layers to detect the different types of thread lines data.

\section{6- Displaying lines and arcs information to the user.}

Fig. 8(a-b) displays the data for all lines and arcs, which are extracted from the part drawing, and will be used in the feature creation module. The extracted data for lines are, start point, end point, and line type (external-internal-thread) line. The extracted data for arcs, start angle, end angle, and line type are displayed.

\section{MANUFACTURING FEATURE CREATION MODULE}

The creation of the manufacturing features means construction of the features geometry and the extraction of the data needed for machining of these features (tolerances, roughness). In order to complete this step, the extracted data will be associated with the created features. This will be discussed in the following steps.

\section{Joining Lines and Arcs to Construct Features}

After reading the data, the software starts to join these lines, and arcs according to certain logic to create the manufacturing features.

\section{Differentiation between External and Internal Features}

The software can distinguish between the created features whether external or internal according to the layer type of the drawing entities, (line and arc) as shown in Fig.8.

\section{Extraction of the Important Points and Dimensions of Feature}

The feature named stepped outer surface shown in Fig.7 in a dark color will be discussed in detail. It consists of two drawing entities (vertical and horizontal lines). 
Figure 9 represents the possible machining sequences for producing this feature. Fig. 9-a represents the machining sequence from maximum diameter to smaller diameter. Fig.9-b represents another machining sequence for producing a stepped outer surface from right to left, by removing; region (1), then region (2) and then region (3) respectively. Region 2 is the most important region, because within this step the required feature will be created. The software will extract all the required data to perform this step. The extracted data has two categories:

1- Points and dimensions belong to feature entities

2- points and dimensions, which are not belong to feature entities.

\section{Extraction of the important points and dimensions related to feature entities}

In Fig.9-a The extracted points, and dimensions for a specific feature are; end point, radial machining allowance $(A / 2)$, and length of cut $(L)$ of the feature, major and minor diameters. All this data is belong to the part drawing entities as follows, the end point lies on the end of the horizontal line. The length of cut is the distance between start and end points of the horizontal line. The major diameter is the distance between the last point on the vertical line and the opposite one, and the minor diameter is the distance between any point on the horizontal line, and the opposite one. Finally the machining allowance is determined from the distance between start and end points of the vertical line of this feature.

In Fig. 9-b the extracted points, and dimensions for a specific feature are start, end points, length of cut, and minor diameter. All this data is belong to the part drawing entities(vertical, and horizontal lines).

\section{Creation of points and dimensions, which are not related to feature entities}

There are some data (points and distances) in certain features which does not exist on the part drawing, and must be extracted to identify these features, so they will be created according to a certain logic as will be discussed in what follows,

As shown in Fig.10 represents the workpiece just perform step for creation the required feature (after removing volume one). In Fig.10-a the extracted points and dimensions are, the start point lies on the extension of the horizontal line in the input part drawing, and length of cut. In Fig.10-b, "Major diameter 1", which is represented by the distance between the two points1 and 2, however these points are not related to the feature entities. The total machining allowance $(\mathrm{A} 1) / 2$, which is represented by the distance between the start point of the horizontal line point 2 and point 3 which is not related to this feature.

\section{Extraction of the Roughness Value}

The surface roughness mark on the part drawing is input in the form of a block, which should be inserted at any point along the line representing the feature This value will be extracted and taken into consideration in the process planning module.

\section{Extraction and Calculation of the IT Grade of Accuracy}

Tolerated dimensions are input as mentioned before in the rules as shown in Fig. 11 for example. The tolerance grade is calculated as follows [12]. Tolerance on the workpiece $\mathrm{T}_{\mathrm{W}}$ is calculated from the upper and lower limits of the given dimension

$\mathrm{T}_{\mathrm{W}}=$ upper limit - lower limit.

Basic tolerance $T_{i}=T_{W} / i_{t}$. 
where $i_{t}=0.45(D)^{1 / 3}+0.001 D$

For the example shown in Fig. 10

$\mathrm{T}_{\mathrm{W}}=70-0=70 \mu \mathrm{m}$.

$\mathrm{i}_{\mathrm{t}}=0.45(80)^{1 / 3}+0.001(80)=2.01 \mu \mathrm{m}$

$\mathrm{T}_{\mathrm{i}}=70 / 2.01=34.67 \mu \mathrm{m}$.

ISO grade: (IT8).

\section{Extraction of Data Required for Thread (Nominal Size, Thread Pitch, etc.)}

The thread has to be instructed on the part drawing in the general form $M$ (nominal size $x$ pitch) as shown in Fig.12. If the user enters $M$ (nominal size) only for threads having a standard pitch, the software will then extract its pitch and hence all other dimensions required for thread machining are determined according to "metric standard for worldwide manufacturing[13]. All previously extracted data for thread will be extracted again for each feature according to its machining requirements.

\section{Displaying Data to the User}

This feature recognition software has an efficient displaying form for the recognized features, and their specific data. Fig.13 displays the output form, which appears to the user after feature creation. As shown there exist two types of features (External \& Internal) .This form contains all features that can be recognized, whereas only the encountered one in the part drawing will be active with dark color, while the other features will remain inactive with grey color. By clicking on the required feature the detailed information about this feature will appear to the user as shown in the Fig.14. By clicking on the graph command button, an example for each feature will appear to identify all data extracted for this feature as shown in Fig. 15.

Some features may be repeated in the same part. this will appear in the feature representation form only once, as shown in Fig.13, but in detail data form each feature will appear with its detail data, as shown in Fig.16 data are displayed for right, and left faces.

\section{Determination of the Size of Raw Material}

The created system can estimate semi product dimensions with machining allowances, radial and longitudinal allowance:

The radial allowance $\left(A_{r}\right)$ given by[14].

$\mathrm{A}_{\mathrm{r}}=0.3 \mathrm{D}_{0}^{0.5} \mathrm{~L}_{0}^{0.125}$.

$\mathrm{D}_{0} \ldots$ nominal diameter of the part

$L_{0} \quad \ldots$ nominal length of part drawing

The longitudinal allowance $\left(A_{l}\right)$ given by

$A_{l}=k_{0}^{0.5} L_{0}^{0.125}$.

$\mathrm{k}=0.2$ for machining on one end face.

$\mathrm{k}=0.3$ for machining on both end faces.

These relationships are applied to a workpiece having a diameter of $70 \mathrm{~mm}$ and a length of $83 \mathrm{~mm}$. The final semi product size is given as shown in Fig.16. 


\section{MACHINING FEATURES}

The most frequently encountered machining features geometry (external and internal) listed in Table 1.

\section{CASE STUDY}

As shown in Fig.17 the part consists of seven external features from right to left (right face - cylindrical - thread - recess - deep stepped outer surface - max outer surface left face). It also includes 6 internal features (Internal chamfer - 2 internal shoulders internal recess - min Internal diameter -internal fillet). Both external and internal features are shown in details in Fig.18, Fig.19. The software extracts the information from the DXF file of the part drawing, and the results must be compatible with the expected feature information. It extracts also the surface roughness values, the tolerance for each feature, and displays these values with the features data. The extracted data is displayed in Fig.13 in dark color. The extracted data is compatible with the predefined features.

\section{CONCLUSION}

This paper includes a complete feature recognition technique which is developed for rotational parts. This technique has been made capable of dealing with DXF file as an input data for any part drawing in full cross sectional view. It is capable also of:

- Extraction of the important lines and arcs data.

- Preparation of the extracted data for creating manufacturing features.

- Joining the prepared data within certain logic to create manufacturing features.

- The developed software can deal with wide range of turning manufacturing features, 16 external, and 10 internal.

- Extraction the important data like dimension, roughness, tolerance, thread pitch, calculate allowance for finishing and relate each value to its specific features.

- Calculation of the size of the required raw material.

\section{REFERENCES}

[1] X.G. Ming, K. L. Mak, and J. Q. Yan " A hybrid intelligent inference model for computer aided process planning" Integrated Manufacturing Systems. 10/6 PP 343-353 (1999).

[2] V. N. Vittal, V. K. Jain and Kripa Shankar " A computer-Aided Process Planning System for rotational parts (CAPP-RP) for an FMS environment" International Journal of Computer Applications in Technology, Vol. 3, No.2, PP 61-69, (1992).

[3] J. Dong, and S. Vijayan "features extraction with the consideration of manufacturing processes." INT. J. Prod. Res., Vol. 35, No. 8,PP 2135-2155, 1997.

[4] Gayankar, P. and Henderson,M.R., Graph-based extraction of protrusions and depressions from boundary representations. computer aided design,1990,22(7), 442450. 
[5] Somashekar Subrahmanyam, and Michael Wonzy "An Overview Of Automatic Feature Recognition Techniques For Computer-Aided Process Planning" computers in industry PP 1-21 (1995).

[6] W. Eversheim, J. Schneewind "Computer-Aided Process Planning-State Of The Art And Future Development" computer-integrated manufacturing, Vol. 10 No. 12 pp. 65-70, 1993.

[7] Charles M., Gerald R., and David A " Computer aided process planning with CMPP “ Computer ind. Eng. Vol.17, Nos1-4, pp 246-251, 1989.

[8] H. B. Marri, A. Gunasekaran, and R. J. Grieve" Computer-Aided Process Planning: A State of Art." Int. J. Adv. Manuf. Technol, vol(14) PP 261-268, (1998).

[9] Eric Wang "Using Automatic Feature Recognition To Interface CAD To CAPP" computers in engineering - Volume 1,PP 215-231, ASME 1992.

[10] R. L. Sakal and J. G. Chow" An Integrated intelligent process planning System for Prismatic parts Using PC-based CAD and CAM Software package" Int. Journal of Advanced manufacturing Technology PP166-174, 1994.

[11] Carlos Rico, Carlos Suarez, Sabino Mateos, Eduardo Cuesta, and Angel Duarte" An Automatic CAPP System for Rotational parts" IEEE1997.

[12] DR. ING. W.LUTZ Maximale Ausspannlange bie, fliegender Bearbietung, und wellenformiger Futterbearbeitung langer Drehwerkstucke, M.aschinemarkt, jg. 74 1968 Nr71, Pp 1390-1393, (1988).

[13] Knut O.kvernelant " metric standard for world wide manufacturing asme press newyork (1996).

[14] EL Hakim M.A."Determination Of The Machining Allowances By The Aid Of Digital Computer "Third conference on statistics, computer science, and social research, PP397-408, (1978). 


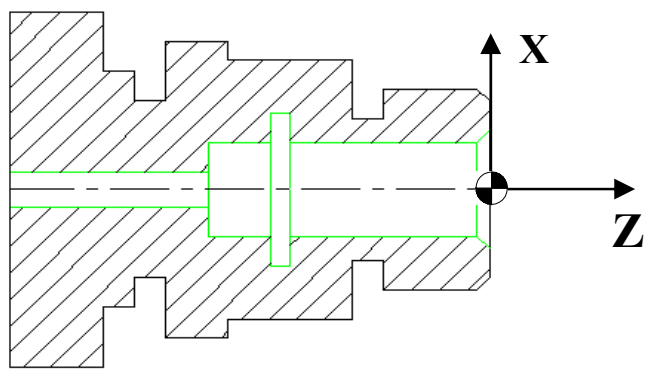

Fig.1. Workpiece zero position

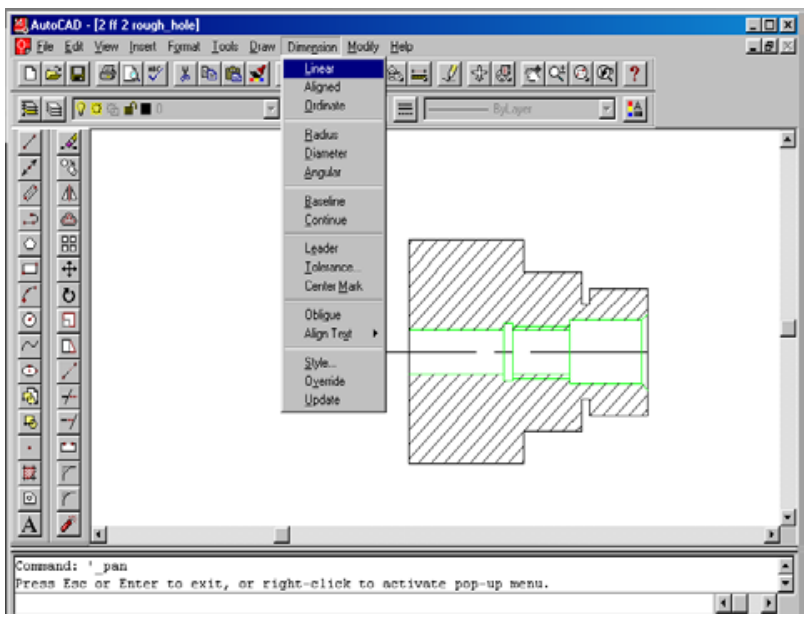

Fig.2 Dimension input method

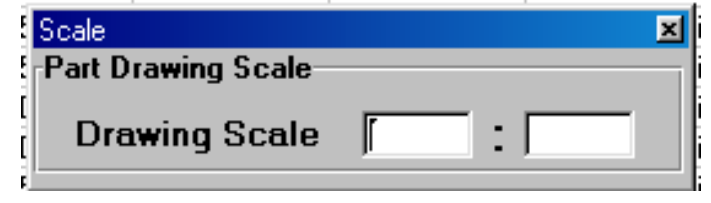

Fig.3 Scale form

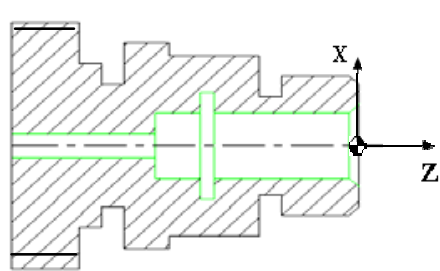

Fig.4. Input part drawing

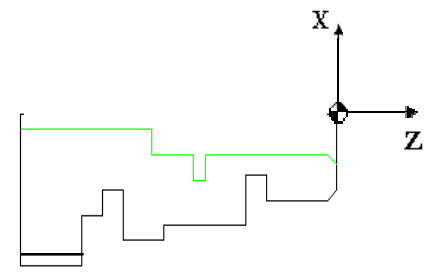

Fig.4-b. Required part drawing
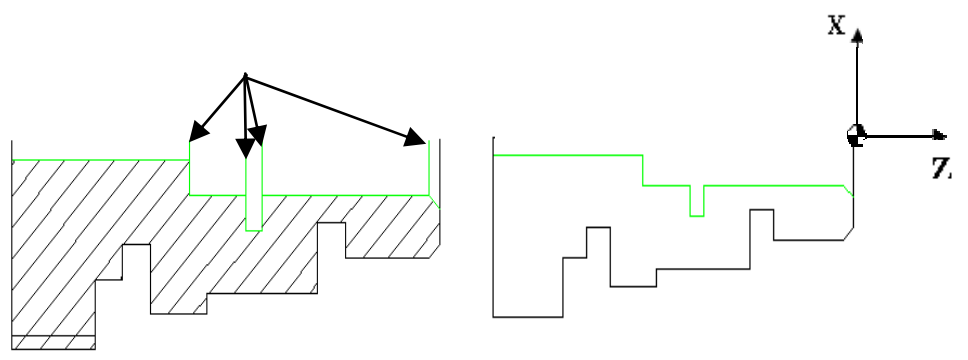

Fig 6 Internal line to be removed

Fig.5 Remove half section

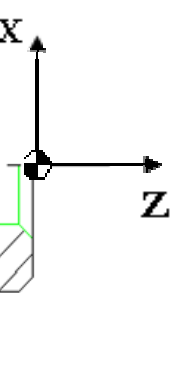




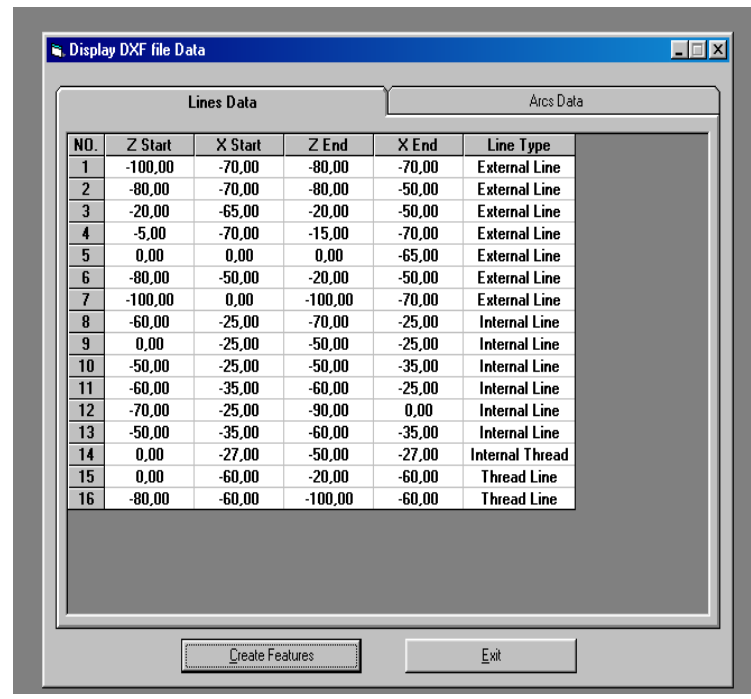

a Display format of extracted lines

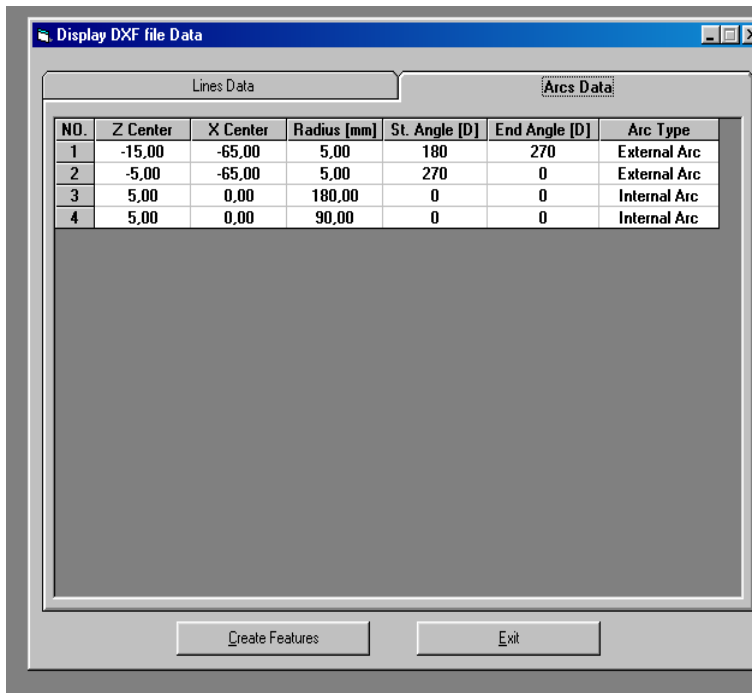

b Display format of extracted arcs

Fig.8

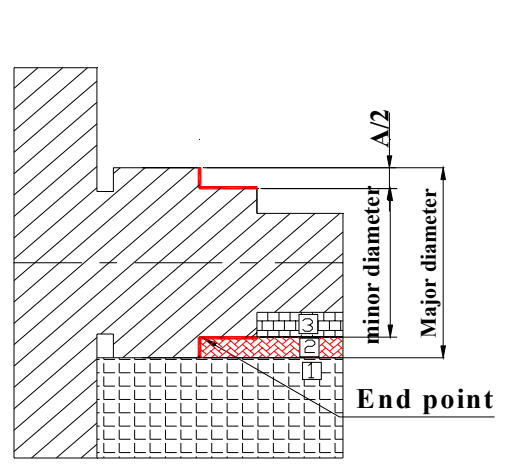

(a)

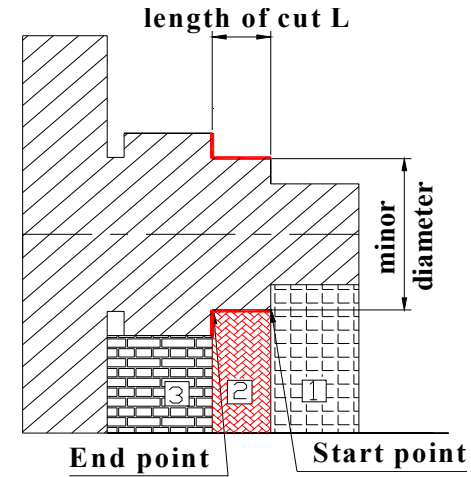

(b)

Fig.9. Extraction of data related to feature entities

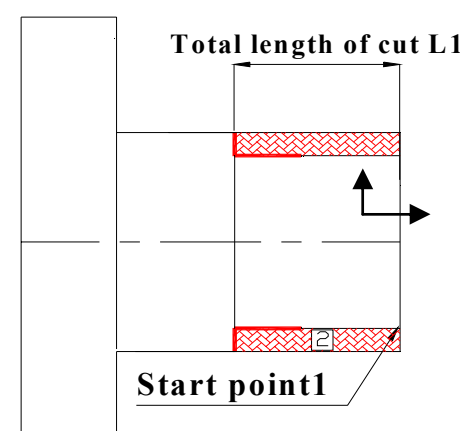

(a)

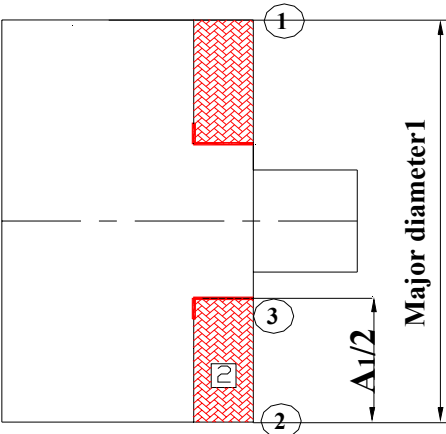

(b)

Fig. 10. Extracted data not related to feature entities 


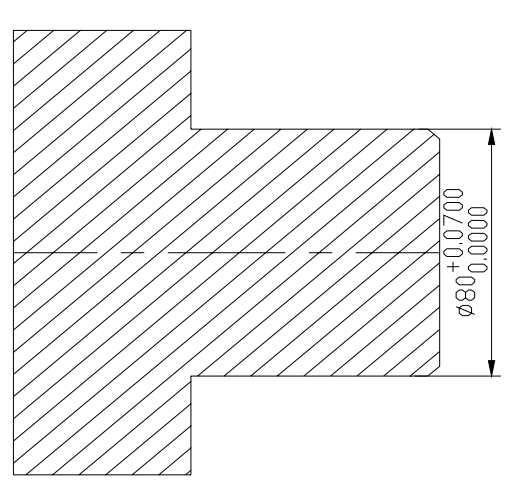

Fig.11. Extraction of tolerance

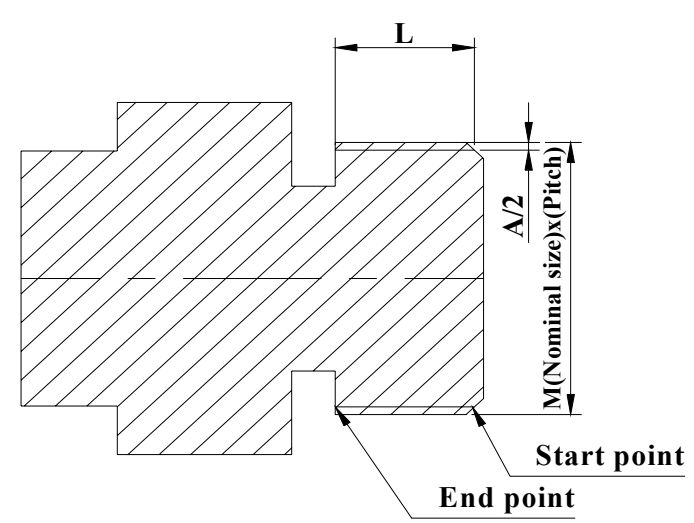

Fig.12. Extracted thread data

\begin{tabular}{|c|c|c|}
\hline \multicolumn{2}{|l|}{ Disply Features Data } & $-\square x$ \\
\hline \multicolumn{2}{|c|}{ EXTERNAL FEATURES } & INTERAL FEATURES \\
\hline Face & Recess & Min. Internal Diameter \\
\hline Chamfer & Fillet & Internal Recess \\
\hline Deep Stepped Outer Surface & Filleted Recess & Internal Thread \\
\hline Thread & Filleted Cylindrical Surface & Internal Shoulder \\
\hline Deep Cylindrical Surface & Filleted Surface & Internal Chamfer \\
\hline Wide Recess & Conical Surface & Internal Cavity \\
\hline Max. Outer Diameter & & Internal Fillet \\
\hline Stepped Outer Surface & & Internal Filleted Recess \\
\hline Stepped Recess & & Internal Filleted Cylindrical \\
\hline Cylindrical Surface & & Internal Filleted Surface \\
\hline
\end{tabular}

Fig.13. Extracted Features

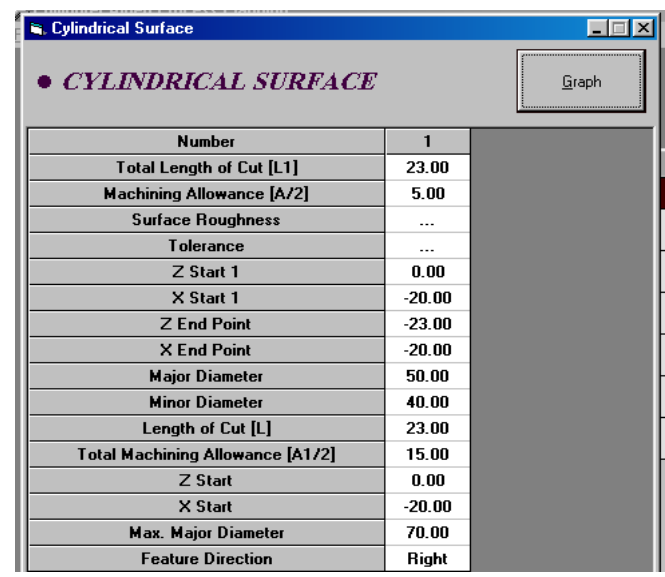

Fig.14. Detailed data form of the cylindrical surface

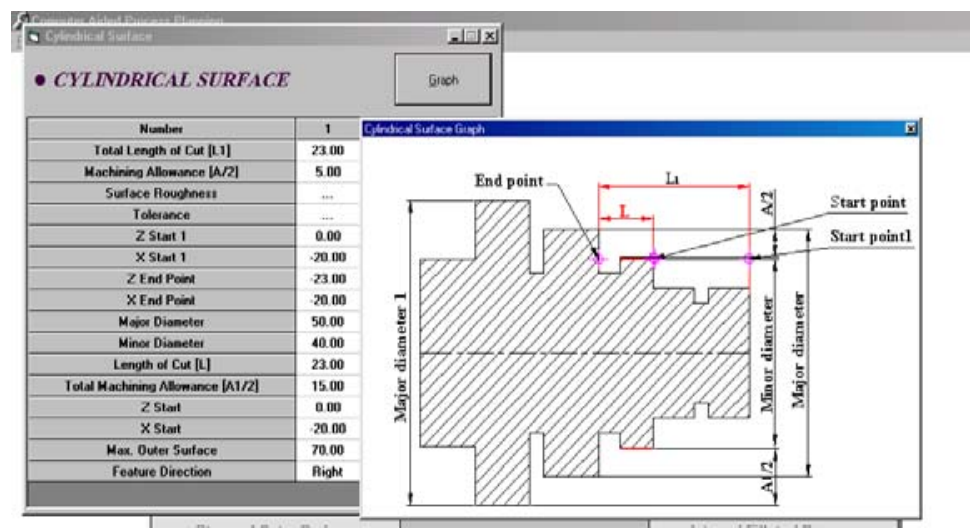

Fig.15. Graphical display of an extracted feature 


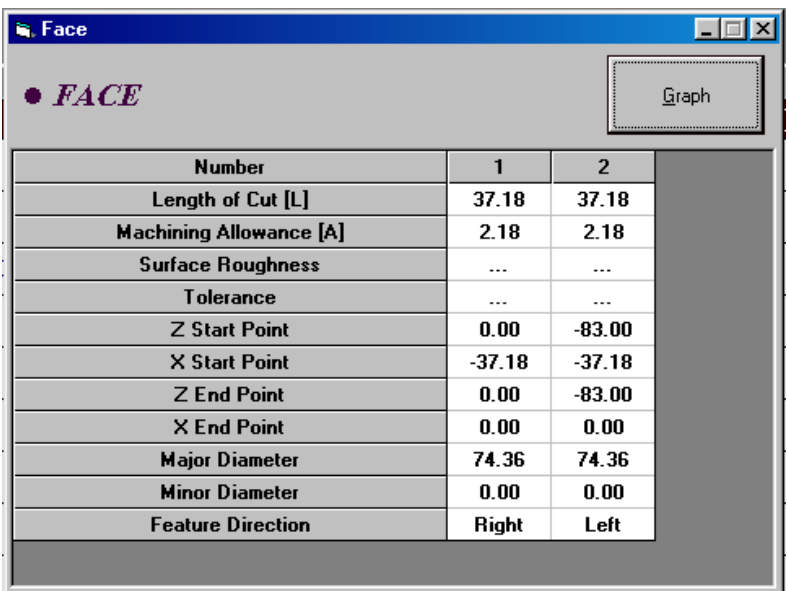

Fig.16. Extraction of data for repeated feature
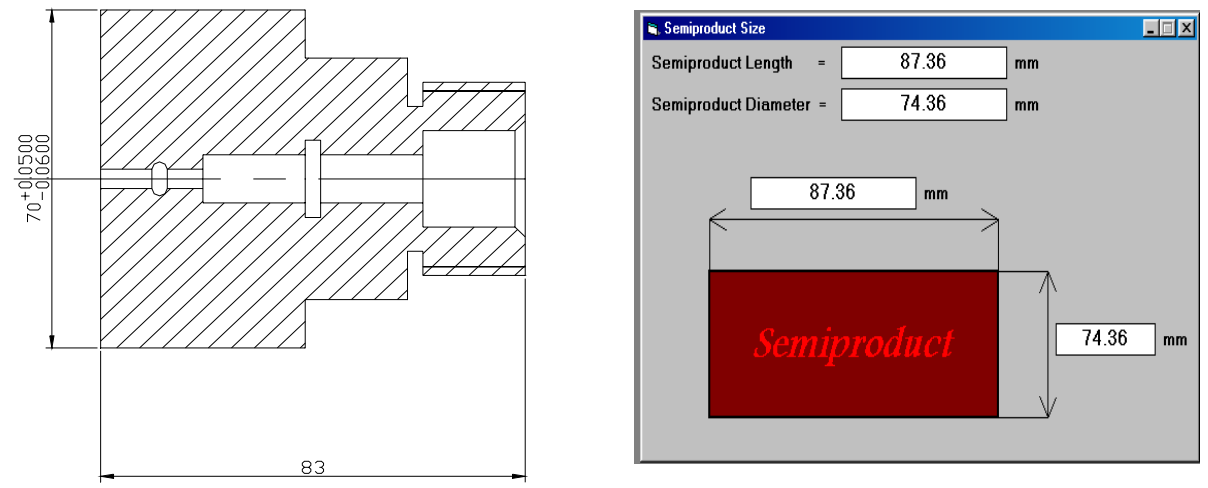

Fig.17. Case study for semiproduct calculation

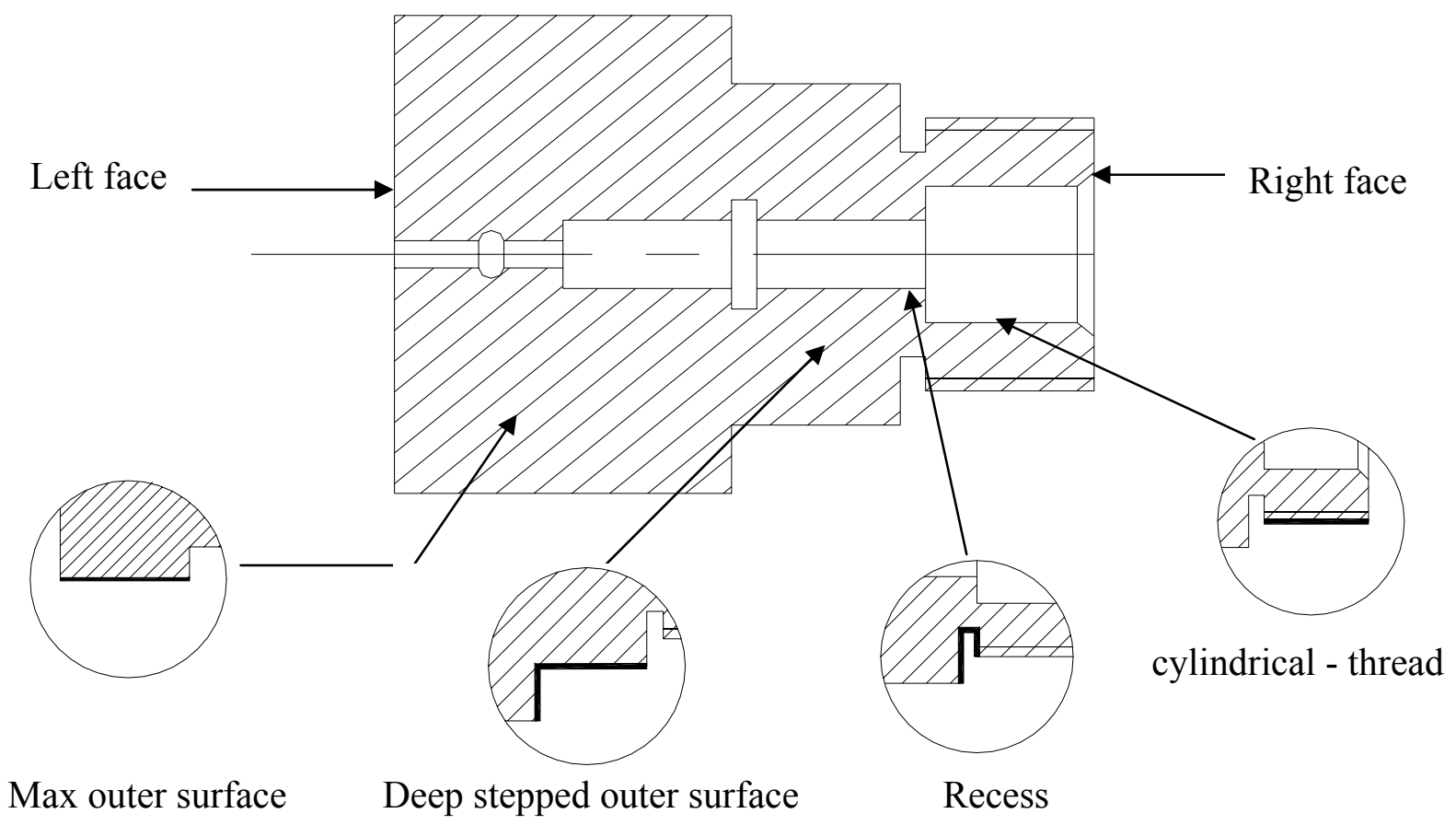

Fig.18. External feature 


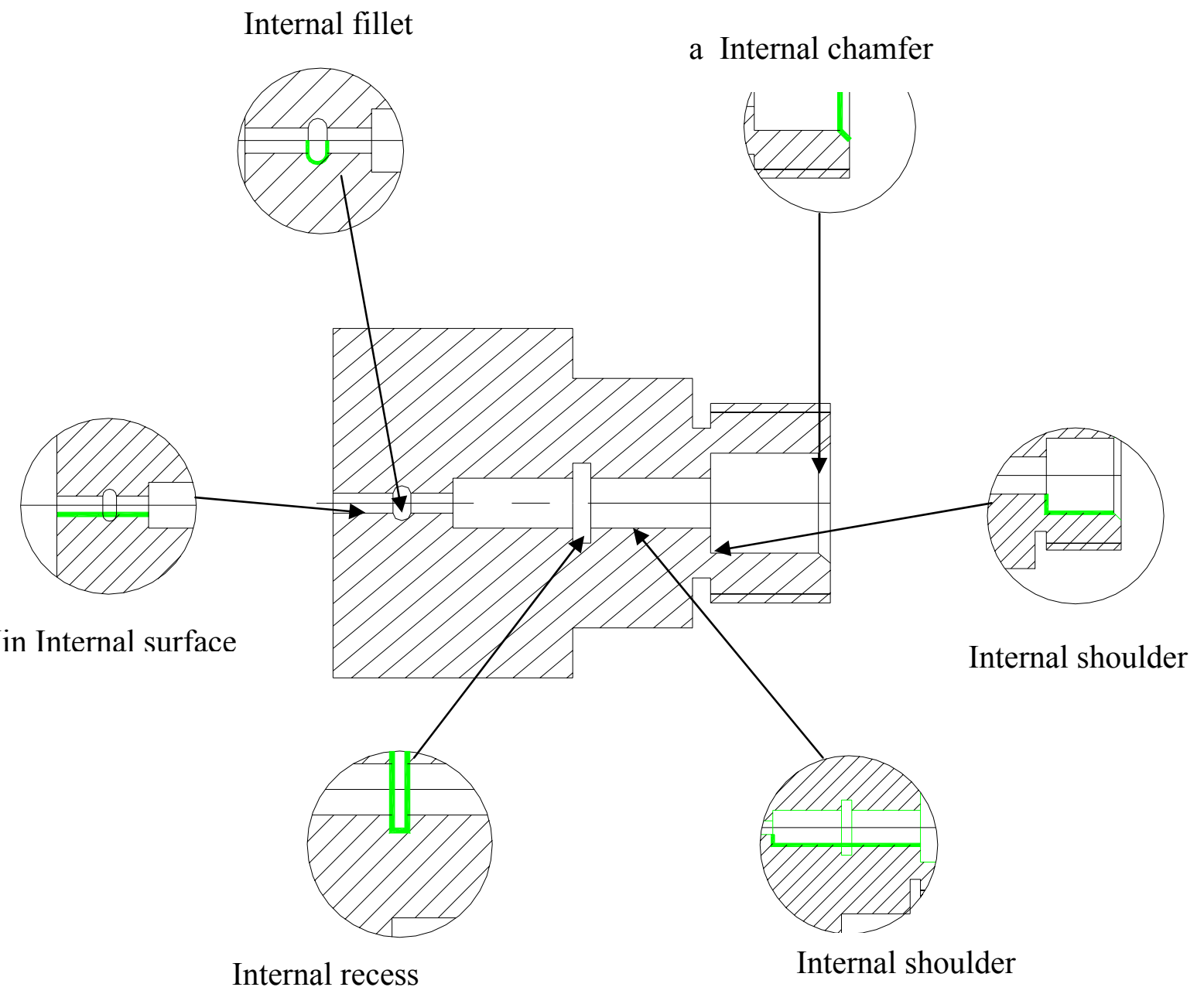

Fig.19. Internal features 
Table 1. List Of External And Internal Manufacturing Features.

\begin{tabular}{|l|l|l|l|}
\hline NO. & External features & NO. & Internal features \\
\hline 1 & Side Face & 1 & Min internal diameter(Hole) \\
\hline 2 & Chamfer & 2 & Int. Chamfer \\
\hline 3 & Recess & 3 & Int. recess \\
\hline 4 & Wide recess & 4 & Int. wide recess \\
\hline 5 & Filleted recess & 5 & Int. filleted recess \\
\hline 6 & Fillet & 6 & Int. fillet \\
\hline 7 & Stepped recess & 7 & Int. stepped recess \\
\hline 8 & Stepped outer surface & 8 & Int. Shoulder \\
\hline 9 & Deep Stepped outer surface & 9 & Int. deep Shoulder \\
\hline 10 & Cylindrical surface & 10 & Int filleted cylindrical surface \\
\hline 11 & Deep Cylindrical surface & & \\
\hline 12 & Filleted cylindrical & & \\
\hline 13 & Filleted surface & & \\
\hline 14 & Taper & & \\
\hline 15 & Max outer surface & & \\
\hline 16 & Thread & & \\
\hline
\end{tabular}

